\title{
Somatic cytochrome $c(C Y C S)$ gene expression and promoter-specific DNA methylation in a porcine model of prenatal exposure to maternal dietary protein excess and restriction
}

\author{
Simone Altmann ${ }^{1}$, Eduard Murani ${ }^{2}$, Manfred Schwerin ${ }^{1}$, Cornelia C. Metges ${ }^{3}$, Klaus Wimmers ${ }^{2}$ \\ and Siriluck Ponsuksili ${ }^{1 *}$ \\ ${ }^{1}$ Research Group 'Functional Genomics', Leibniz Institute for Farm Animal Biology, Wilhelm-Stahl-Allee 2, D-18196 \\ Dummerstorf, Germany \\ ${ }^{2}$ Research Unit 'Molecular Biology', Leibniz Institute for Farm Animal Biology, Wilhelm-Stabl-Allee 2, D-18196 \\ Dummerstorf, Germany \\ ${ }^{3}$ Research Unit Nutritional Physiology 'Oskar Kellner', Leibniz Institute for Farm Animal Biology, Wilhelm-Stabl-Allee 2, \\ D-18196 Dummerstorf, Germany \\ (Received 6 January 2011 - Revised 6 June 2011 - Accepted 6 June 2011 - First published online 1 September 2011)
}

\section{Abstract}

There is growing evidence that maternal nutrition during gestation has an important effect on offspring development as well as on their gene expression with long-term effects on the metabolic state. A potential mechanism forming long-lasting gene expression patterns is DNA methylation of cytosine in $\mathrm{CPG}$ dinucleotides within the promoter region of distinct genes. There has been special focus on mitochondrial dysfunction by prenatal malnourishment over the recent years. To this end, we investigated the gene expression of somatic cytochrome $c$ (CYCS), an important member of the respiratory chain, in a porcine model of gestational protein over- and undersupply at $94 \mathrm{~d}$ post-conception and 1, 28 and $188 \mathrm{~d}$ of age, and analysed the association with the DNA methylation status within the CYCS promoter. Gene expression on day 1 post natum showed a significant increase in the low protein (LP) group $(P=0 \cdot 0005)$ and a slight increase in the high protein (HP) group $(P=0.079)$ compared with the control (CO) group in the liver. The mean of the methylation level over forty-seven CpG sites from nucleotide (nt) -417 to -10 was significantly decreased in the LP $(P=0 \cdot 007)$ and HP $(P=0 \cdot 009)$ groups compared with that in the CO group. Excess and restricted protein supply during pregnancy led to hypomethylation of a number of CpG sites in the CYCS promoter, including those representing putative transcription factor-binding sites, associated with elevated expression levels. However, the impact of the low-protein gestation diet is more pronounced, indicating that the offspring could better adapt to excess rather than restricted protein supply.

Key words: Protein restriction: High-protein diet: Gestation: Maternal diet: Fetal programming: Pigs: Intra-uterine growth restriction

In rodent models and in humans, the impact of gestation diets on the offspring's phenotype was shown experimentally and epidemiologically. The underlying programming of fetal development is seen as a risk factor for degenerative diseases in adulthood, including the metabolic syndrome. The fetal programming of adult disease hypothesis has been summarised $^{(1-3)}$. This relationship was considered as a result of fetal adaptations to intra-uterine malnutrition leading to decreased birth weight and a higher risk for adult disease, which, in the case of a discrepancy between fetal and adult nutrition, leads to increased sensitivity to metabolic disorders ${ }^{(4)}$.
In recent years, there has been increasing interest in intrauterine programming of traits through maternal nutrient deficiency or excess in livestock ${ }^{(5,6)}$. Among the livestock species, pigs most prevalently suffer from intra-uterine growth retardation as a result of deficient fetal nutrient supply ${ }^{(5,6)}$. Piglets with below average birth weight develop reduced muscle growth but increased fat deposition, resulting in impaired meat quality traits with tremendous effects on farm animal production and welfare ${ }^{(7)}$.

There is evidence that intra-uterine malnutrition can have long-lasting effects on the gene expression of the fetus and

Abbreviations: CO, control; dpn, dies post natum; HP, high protein; LP, low protein; NRF-1, nuclear respiratory factor 1; nt, nucleotide; TFBS, transcription factor-binding sites.

*Corresponding author: Dr S. Ponsuksili, fax + 4938208 68-702, email s.wimmers@fbn-dummerstorf.de 
therefore can extensively affect the phenotypic outcome of the progeny ${ }^{(8,9)}$. In rodents, gestational protein restriction is a well-established animal model for the study of nutrientgene interactions in the offspring ${ }^{(10,11)}$. Epigenetic effects manifesting during crucial pre- and perinatal developmental stages are increasingly seen as underlying effects of fetal programming ${ }^{(12)}$. Epigenetic effects involve molecular mechanisms leading to heritable changes in gene expression without nucleotide (nt) sequence alterations ${ }^{(13)}$. DNA methylation is a well-investigated mechanism of epigenetic gene regulation in which a methyl group is transferred to the $5^{\prime}$ position of genomic cytosine in CPG dinucleotides by DNA methyltransferases ${ }^{(14)}$. CPG dinucleotides are not equally distributed throughout the vertebrate genomes but accumulate in CpG-rich areas of DNA termed as CpG islands ${ }^{(15,16)}$. CpG islands are usually hypomethylated and associated with the promoter regions of protein-coding genes ${ }^{(17)}$.

The consequences of maternal protein restriction on the mitochondrial function of the offspring were recently described in rodent models suggesting a role for nutritional mitochondrial programming in the development of mitochondrial dysfunction ${ }^{(18,19)}$. The process of oxidative phosphorylation taking place in the mitochondria is an important part of energy metabolism and is essential for ATP synthesis. Somatic cytochrome $c$, encoded by CYCS, is an integral membrane protein occurring in the mitochondrial intermembrane. It acts as an electron transfer protein in the respiratory chain of oxidative phosphorylation transferring one electron from cytochrome bc1 complex to cytochrome $c$ oxidase. Cytochrome $c$ is also involved in apoptosis when released into the cytosol as a coactivator for caspase- 3 activation because it binds the apoptotic protease activating factor 1 thus activating downstream effector caspases ${ }^{\text {(20-23). }}$.

Based on its function in cell energy metabolism as well as an apoptotic marker, CYCS was chosen as a candidate gene to investigate the influence of gestational protein restriction on mRNA expression and its relationship with the methylation status of the promoter-associated $\mathrm{CpG}$ island in a porcine model.

\section{Experimental methods}

\section{Animals, diets and sample collection}

The animal care and tissue collection processes followed the guidelines of the German Law of Animal Protection, and the experimental protocol was approved by the Animal
Care Committee of the State Mecklenburg-Vorpommern (Germany). The animal experiment was performed as described previously ${ }^{(24)}$. In brief, primiparous German Landrace (DL) sows were inseminated at the age of 8 months. The gilts were randomly assigned at mating to the three diet groups and fed these diets throughout gestation: control (CO) group containing $12 \cdot 1 \%$ crude protein (\% of DM); high protein (HP) group with $30 \%$ crude protein; low protein (LP) group containing $6.5 \%$ crude protein, corresponding to a protein:carbohydrate ratio of $1: 5,1: 1 \cdot 3$ and $1: 10 \cdot 4$, respectively. The diets were formulated to be isoenergetic (13.6 MJ metabolisable energy/kg) ${ }^{(24)}$. Within $36 \mathrm{~h}$ after birth, the piglets were cross-fostered by two to four parity sows that were fed a standard diet during pregnancy. During lactation, a single standard lactation diet was fed (Provital LAC R.325.0; Trede \& von Pein, Dammfleth, Germany) with 15.5\% crude protein, $0.95 \%$ lysine, approximately $13.0 \mathrm{MJ}$ metabolisable energy/kg. After weaning at the age of $28 \mathrm{~d}$, the offspring were fed ad libitum a standard diet for growing and finishing pigs until the end of the experiment (age 185d). In the present study, the liver samples of offspring derived from a subset of sows of the three diet groups were collected at gestational day 94 ( $n_{\text {offspring }}$, sixteen per diet group), at birth (dies post natum

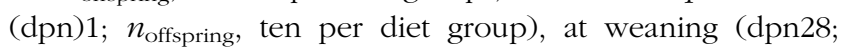

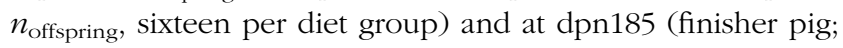

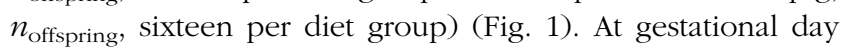
94, the fetuses were developed from six sows per diet group by caesarean section. All the liver samples were immediately frozen in liquid $\mathrm{N}_{2}$ and stored at $-80^{\circ} \mathrm{C}$ until DNA and RNA extraction. Husbandry and slaughtering conditions have already been described in detail ${ }^{(25)}$.

The body weights at gestational day 94 of the fetuses analysed in the present study did not differ among the three diet groups. However, when scoring all the fetuses derived from the complete experiment, HP and LP offspring were growth restricted compared with the CO offspring, but litter size did not differ ${ }^{(24)}$. At birth, the piglets of the LP and HP groups were significantly lighter than the piglets of the $\mathrm{CO}$ group $(1.22$ (SEM 0.05$) \mathrm{kg}$ and LP, 1.06 (SEM 0.05$) \mathrm{kg}$; $P=0.033$ ); also piglets of the HP group were lighter than the piglets of the CO group, but that differences were not significant for the animals used in this analysis. Taking into account all the animals of the experiment, the HP and the LP diets led to significant growth retardation at dpn1. This difference did not persist until weaning at dpn28. Also, finisher pigs at the

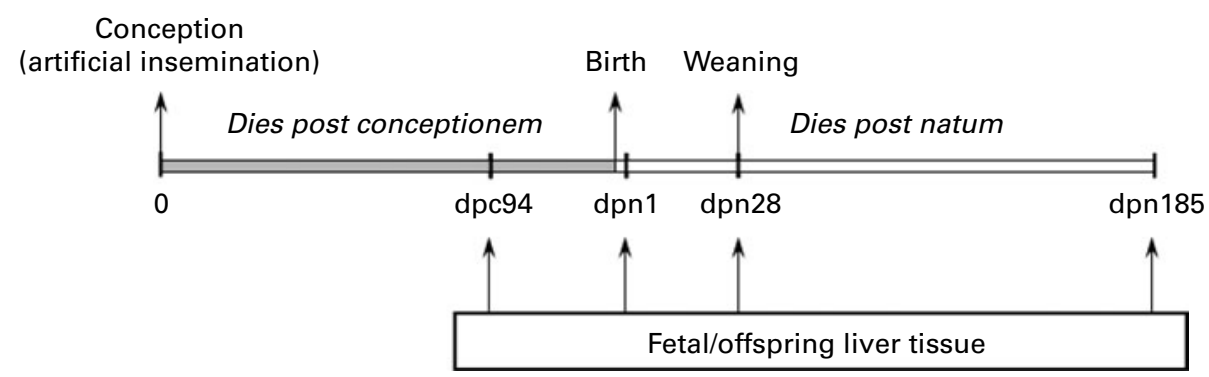

Fig. 1. Time bar of the experimental procedure. Nullipara gilts were artificially inseminated (day 0). Samples of offspring liver tissue were taken at conceptional day 94 as well as at postnatal days 1,28 and 185. dpc, Dies post conceptionem; dpn, dies post natum. 
age of $185 \mathrm{~d}$ showed no significant differences in body weight dependent on the diet group ${ }^{(25)}$.

\section{DNA and RNA isolation}

The liver samples were powdered by mortar and pestle in liquid $\mathrm{N}_{2}$. For DNA isolation, phenol-chloroform extraction was performed using Eppendorf Phase Lock Gel Tubes (Eppendorf, Hamburg, Germany). After precipitation, the DNA pellets were dissolved in $500 \mu$ l Tris-EDTA (TE) buffer, and concentration and quality were checked by a spectrophotometer (ND1000; Nano Drop Technologies, Wilmington, DE, USA). DNA was stored at $-20^{\circ} \mathrm{C}$.

RNA was isolated by TRIzol, phenol-chloroform extraction and cleaned with the RNeasy Mini Kit and the RNase-Free DNase Set (Qiagen GmbH, Hilden, Germany) following the supplier's protocol. RNA was quantified spectrophotometrically and stored at $-80^{\circ} \mathrm{C}$.

\section{$R T$ and quantitative real-time $P C R$}

First-strand complementary DNA was synthesised from $2 \mu \mathrm{g}$ of total RNA with random primers (Fermentas GmbH, St LeonRot, Germany), oligo d(T) 13VN (Sigma-Aldrich, Munich, Germany) and the SuperScript ${ }^{\mathrm{TM}}$ II Reverse transcriptase Kit (Invitrogen, Darmstadt, Germany) following the manufacturer's guide. Real-time quantitative PCR was performed using the LightCycler ${ }^{\circledR} 480$ Real-Time PCR System (Roche Diagnostics GmbH, Grenzach-Wyhlen, Germany). The amplification was conducted in duplicate according to the supplier's instructions. The reactions were performed in a final volume of $10 \mu \mathrm{l}$ using $5.0 \mu \mathrm{l}$ of LightCycler 480 SYBR Green I Master (Roche Diagnostics GmbH), $2 \cdot 0 \mu$ l of Aqua dest, $10 \mu \mathrm{m}$ of each primer and $40 \mathrm{ng}$ of complementary DNA. The temperature profiles comprised an initial denaturation step at $95^{\circ} \mathrm{C}$ for $10 \mathrm{~min}$ and forty cycles consisting of denaturation at $95^{\circ} \mathrm{C}$ for $15 \mathrm{~s}$, annealing at $60^{\circ} \mathrm{C}$ for $10 \mathrm{~s}$ and extension at $72^{\circ} \mathrm{C}$ for $15 \mathrm{~s}$. After the completion of the amplification protocol, all samples were subjected to melting curve analysis and gel electrophoresis. For all the assays, the threshold cycles were converted to copy numbers using a standard curve generated by amplifying the serial dilutions of an external PCR standard $\left(10^{2}-10^{7}\right.$ copies). The primers were obtained from Sigma-Aldrich. The primer sequences for CYCS are given in Table 1. Normalisation of the variation in RT-PCR efficiency and initial RNA input was performed using RPL32 and RPL1O genes as internal standards by dividing the calculated mRNA copy numbers by a mean normalisation factor derived from the expression of the reference genes (transcript amount).

\section{Porcine CYCS promoter analysis and $C P G$ island identification}

The genomic sequence of porcine CYCS was obtained from Ensembl Pig FPC map and NCBI nucleotide database and GenBank: FP236676•1_00 902; NC_010460·1 (www.ncbi.nlm.nih.gov; accessed November 2010). For multiple alignment of the CYCS $5^{\prime}$ upstream region, the following genomic sequences of human (GenBank: NC_000007·13), bovine (GenBank: NC_007302.4), canine (GenBank: NC_006596.2) and murine (GenBank: NC_000072.5) species were taken from the NCBI nucleotide database (http://www.ncbi.nlm.nih.gov/; accessed July 2010) and aligned using Mulan ${ }^{(26)}$. The $5^{\prime}$-flanking region was checked for $\mathrm{CpG}$ islands and putative transcription factor-binding sites (TFBS) using EMBOSSs CpGPlot ${ }^{(27)}$ and MultiTF software tools ${ }^{(26)}$, respectively.

\section{Bisulphite sequencing of porcine CYCS promoter}

Genomic DNA from newborn piglets (dpn1) was treated with bisulphite by the EZ DNA Methylation Gold Kit (Zymo Research Corporation, Irvine, CA, USA) according to the supplier's instructions. The converted DNA was amplified by semi-nested PCR. The primers that are used are given in Table 1. Nested PCR was performed as follows using AmpliTaq Gold ${ }^{\circledR}$ DNA Polymerase (Applied Biosystems, Darmstadt, Germany): First PCR: $94^{\circ} \mathrm{C}$ at $4 \mathrm{~min} ; 94^{\circ} \mathrm{C}$ at $30 \mathrm{~s}, 56^{\circ} \mathrm{C}$ at $40 \mathrm{~s}$, $72^{\circ} \mathrm{C}$ at $1 \mathrm{~min} \times 40 ; 72^{\circ} \mathrm{C}$ at $8 \mathrm{~min}$. Second PCR: $94^{\circ} \mathrm{C}$ at $4 \mathrm{~min} ; 94^{\circ} \mathrm{C}$ at $30 \mathrm{~s}, 58^{\circ} \mathrm{C}$ at $40 \mathrm{~s}, 72^{\circ} \mathrm{C}$ at $1 \mathrm{~min} \times 40 ; 72^{\circ} \mathrm{C}$ at $8 \mathrm{~min}$. The PCR products were purified with the QIAquick PCR Purification Kit (Qiagen GmbH). The extent of methylation was determined by direct sequencing of PCR fragments (amplified by using bisulphite-treated DNA as a template) by Qiagen $\mathrm{GmbH}$ as follows: all sequencing reaction mixes were based on the BigDy 3.1 Terminator chemistry (Applied Biosystems). Template amounts of $5 \mathrm{ng} / 100$ bases of fragment length and $10 \mathrm{pmol}$ primer/reaction were used.

Table 1. List of primer pairs used for sequencing, quantitative real-time (RT) PCR and bisulphite direct sequencing (BSP)

\begin{tabular}{|c|c|c|c|}
\hline Primer & $5^{\prime}-3^{\prime}$ sequence & Amplicon (bp) & GenBank resource \\
\hline CYCS-RT-FP & TCСССТССТАСАGAGATGGTT & & \\
\hline CYCS -RT-RP & ATGAGATAGCAAAGGGATCGT & 165 & NM_001129970.1 \\
\hline RPL32-RT-FP & AGCCCAAGATCGTCAAAAAG & & \\
\hline RPL32-RT-RP & TGTTGCTCCCATAACCAATG & 165 & NM_001001636.1 \\
\hline RPL10-RT-FP & CTGTGTTCGTCTTTTCTTCC & & \\
\hline RPL10-RT-RP & TCATCCACTTTTGCCTTCT & 199 & NM_001044543.1 \\
\hline CYCS -BSP-FP1 & TATAAATGATAGGAGAGGGAAGG & & \\
\hline CYCS -BSP-RP1 & СССТСААААСТТААСТТССС & 894 & FP236676.2 \\
\hline CYCS -BSP-FP2 & AGTTTTAGGTGTGTTTAAGTTGG & & \\
\hline CYCS -BSP-RP2 & СССТСААААСТТААСТТССС & 581 & FP236676.2 \\
\hline
\end{tabular}

$\mathrm{FP}$, forward primer; RP, reverse primer. 
The reactions were cycled in a GeneAmp PCR System 9700 (Applied Biosystems) and purified using DyeEx ${ }^{\mathrm{TM}}$ (Qiagen GmbH). Data collection was carried out on a 3730xl DNA Analyser (Applied Biosystems) equipped with $50 \mathrm{~cm}$ capillary arrays and POP $7^{\mathrm{TM}}$ polymer. After data collection, the raw data channels from the result files that were generated (.ab1) were processed using custom-built software developed at Qiagen GmbH. Data processing involved compensation of the different migration properties of the four dyes: baseline correction, peak detection and base calling. For each base, the area was calculated from the corresponding peak to measure the base's quantity. Bases that corresponded to variable positions of $\mathrm{CpG}$ sites were identified by comparison of the obtained sequence with a reference sequence. The ratios of methylated $v$. unmethylated species $(\mathrm{C} / \mathrm{T}$ in forward reads and $\mathrm{G} / \mathrm{A}$ in reverse reads) were reported as percentage methylation. The values of the signal:background ratio ( $\mathrm{S}: \mathrm{B})$ were used as a quality control of quantification. S:B ratios of 20 and above were considered as high-quality samples and used for the quantification of the methylation status. Furthermore, in order to validate the procedure of measurement of the proportion of methylation at the various CpG sites within each fragment - including the steps of bisulphite treatment, direct sequencing and evaluation of electropherograms - non-methylated and 100\% methylated DNA samples were generated. For the generation of unmethylated samples (0\%), genomic DNA was amplified using REPLI-g UltraFast Mini Kit (Qiagen $\mathrm{GmbH}$ ) and purified by phenol-chloroform extraction. To produce $100 \%$ methylated samples, genomic DNA was treated with the CpG Methyl transferase Kit (M.SssI 20000 units/ml; New England Biolabs, Inc., Frankfurt,
Germany) and purified by the phenol-chloroform extraction. Then, the purified 0 and 100\% methylated DNA were treated with bisulphite and used for bisulphite-specific PCR as described earlier. The amplicons of both the treated DNA samples were mixed in different ratios (10-90\%) for direct sequencing by Qiagen Sequencing Services. The algorithm was developed and the procedure was previously validated $^{(28,29)}$

\section{Statistical analyses}

Statistical analyses were performed with SAS 9.2 software package for Windows (SAS Institute, Inc., Cary, NC, USA). Differences in the level of expression of CYCS at each time point, dies post conceptionem 94, dpn1, dpn28 and dpn185, measured by real-time RT-PCR and differences in the level of DNA methylation at dpn1 measured by bisulphite sequencing between samples of the offspring of the respective diet groups were evaluated by ANOVA. The 'general linear models' procedure (SAS Institute, Inc.), i.e. the method of least squares to fit the general linear models, and a post boc Tukey-Kramer multiple comparison procedure were used. Dietary group (CO, HP and LP), class of birth weight (light and heavy), sex (female and male) and sow nested within the diet group were considered as fixed effects. Weight at tissue collection was included as a co-variable. Statistical dependence between methylation status and real-time PCR data was estimated using Spearman's rank correlation coefficient. Values are given as least squares means with their standard errors. A $P$ value of $<0.05$ was considered to be statistically significant.

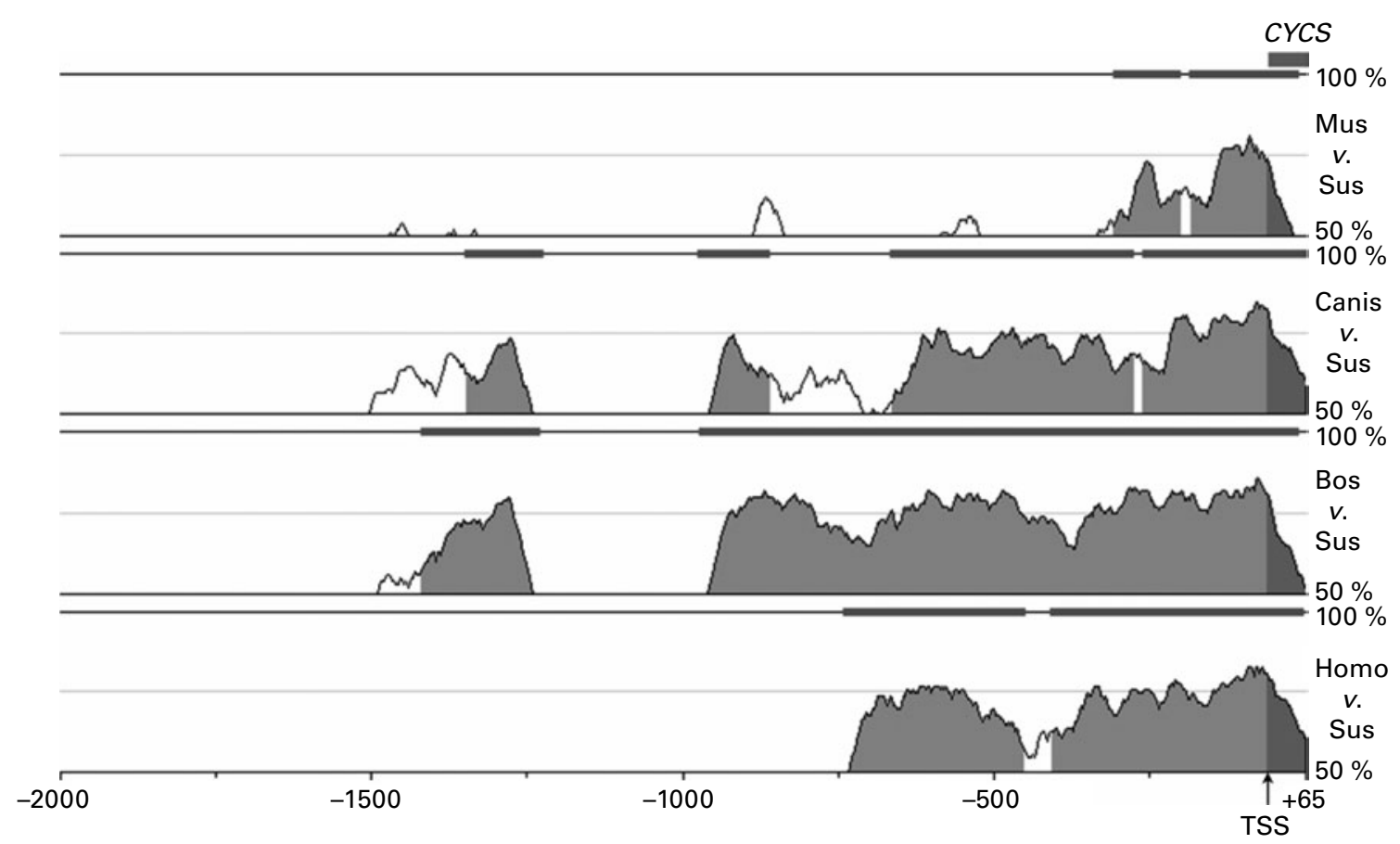

Fig. 2. The Mulan sequence conservation profile for porcine, human, bovine, canine and murine $5^{\prime}$-flanking region of $C Y C S$. The horizontal axis displays the input sequences. Evolutionary conserved regions were detected using a threshold of at least $70 \%$ identity over 100 bp and are depicted as dark bars above each pairwise alignment. The $5^{\prime}$-untranslated region (nucleotide (nt) -2000 to nt +65 ; dark grey) is presented. The $y$-axis shows the percentage similarity of porcine $5^{\prime}$-flanking CYCS orthologue to human, bovine, canine and murine sequences. TSS, transcription start site; Mus, murine; Sus, porcine; Canis, canine; Bos, bovine; Homo, human. 


\section{Results}

Comparative sequence analysis of putative porcine CYCS promoter and $\mathrm{CPG}$ island identification

A 2065 bp fragment of $5^{\prime}$-flanking region including exon 1 (nt -2000 to +65 relative to transcription start site) of porcine CYCS was aligned to its human, bovine, canine and murine orthologues. The multiple alignment analysis revealed the presence of evolutionary conserved regions with $>70 \%$ identity over $100 \mathrm{bp}$. The Mulan sequence conservation profile is represented in Fig. 2. The $5^{\prime}$-upstream region in pigs showed high identity compared with the human, bovine, canine and murine sequences (Fig. 2). Pairwise comparison revealed the bovine sequence as the closest potential evolutionary orthologue to the pig 5'-flanking region supported by the highest pairwise identity and length of evolutionary conserved regions. A MultiTF analysis of bovine and porcine orthologue revealed six multi-conserved specific protein 1 and activator protein 2 TFBS (5'-upstream region from nt -500 to -1 ; Fig. 3). A CpG plot of a DNA region ranging from nt -2000 to +2300

(a)

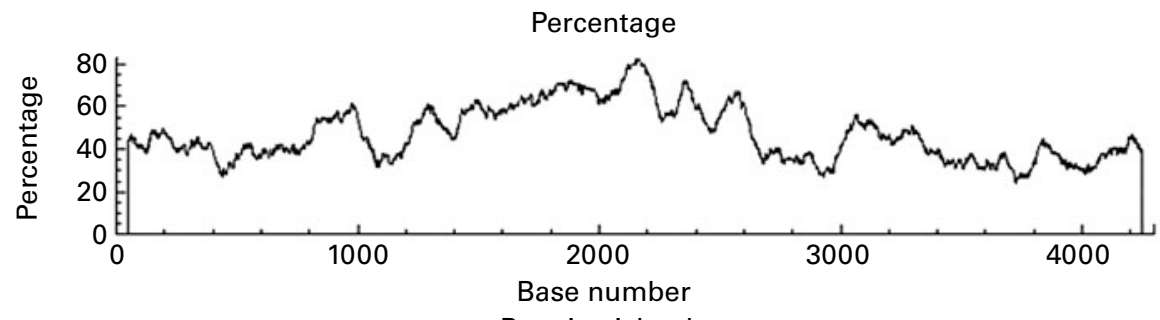

(b)

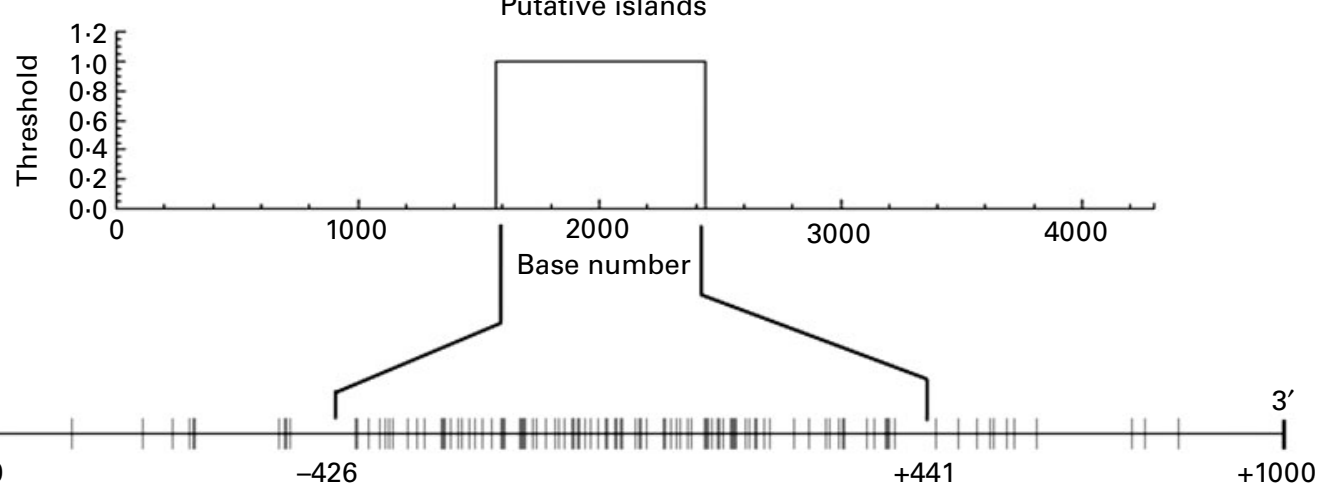

(c)

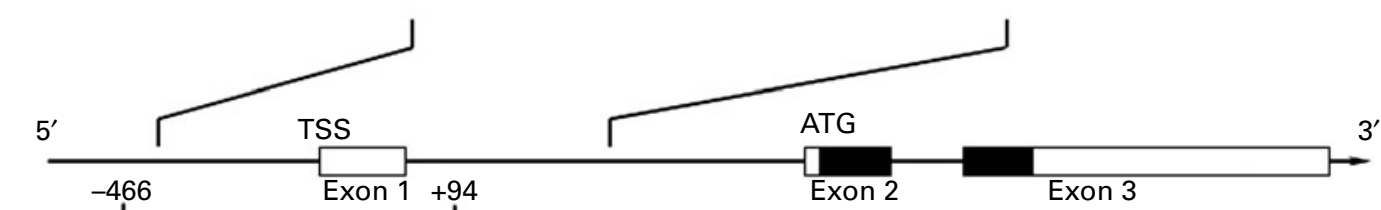

(d)
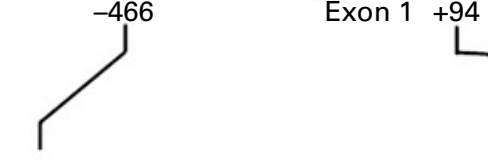

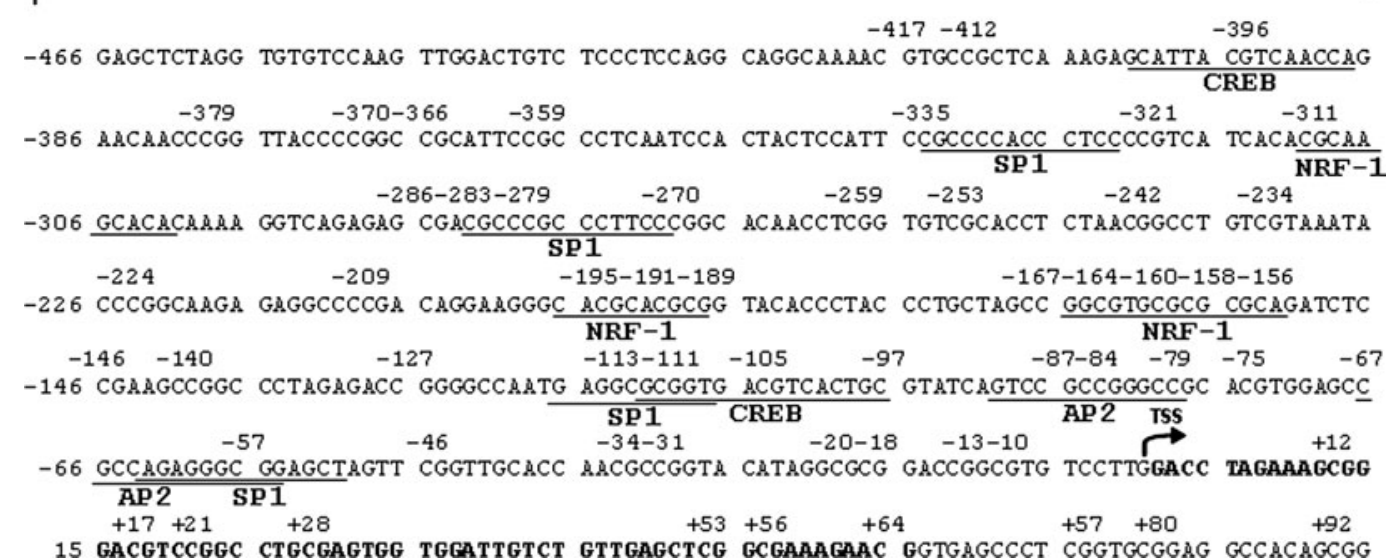

Fig. 3. (a) CpG island identification in the porcine CYCS gene (ENSSSCG00000016714) using EMBOSS CpGPlot (settings: observed:expected ratio $>0.60$, percentage $C$ + percentage $G>60.00$, length $>500$ ). (b) CpG density report, CpG sites are indicated by grey bars. (c) Structure of the porcine $C Y C S$ gene; white boxes, $5^{\prime}$-untranslated region; black boxes, coding sequence; TSS, transcription start site; ATG, start codon. (d) Nucleotide sequence of the investigated part of the CpG island showing individual CpG sites and putative transcription factor-binding sites (MultiTF); SP1, specific factor 1/GC-Box-factor; AP2, activator protein 2; CREB, cAMP response element-binding protein; NRF-1, nuclear respiratory factor 1. 


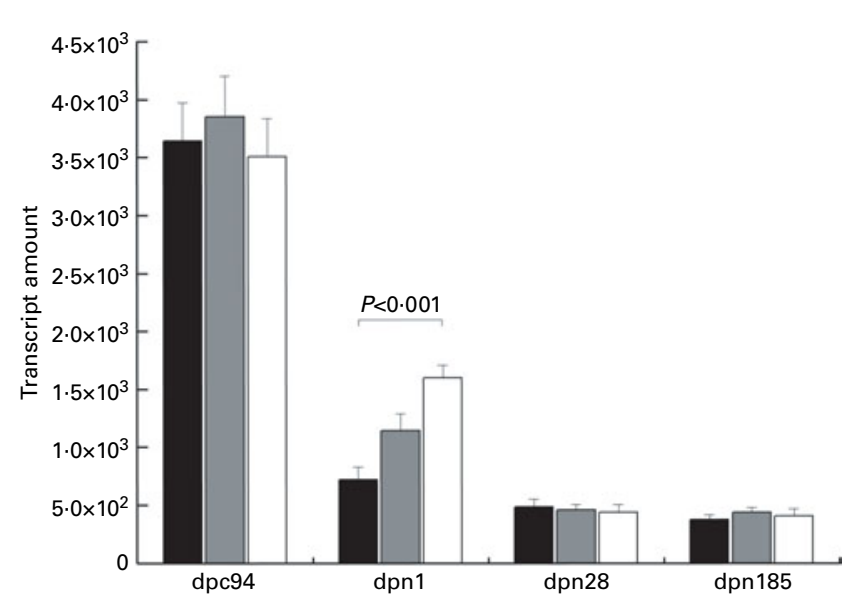

Fig. 4. Transcript amounts of CYCS in the liver at dies post conceptionem (dpc)94, dies post natum (dpn)1, dpn28 and dpn185. Values are least square means, with their standard errors represented by vertical bars. CO ( $\boldsymbol{\square})$, control; HP ( $\square)$, high protein; LP ( $\square)$, low protein.

comprising the whole porcine CYCS sequence showed a 867 bp CpG island from nt -426 to +441 that includes exon 1 (Fig. 3). The schematic structure of the CYCS gene and position of the $\mathrm{CpG}$ island are given in Fig. 3. The CpG island includes ninety-one $\mathrm{CpG}$ sites (10.59\% CpG) and shows an overall $\mathrm{C}+\mathrm{G}$ content of $65.74 \%$ as well as an observed:expected ratio of $0 \cdot 97$

\section{Gene expression of CYCS}

The results of quantitative gene expression analyses are shown in Fig. 4. At gestational day 94, hepatic gene expression of CYCS showed no significant differences among the CO, HP and LP groups. In 1-d-old piglets (dpn1), a protein restriction during pregnancy (LP) resulted in a significantly higher gene expression level in the hepatic tissue compared with the CO group $(P=0.0005)$. The abundance of $C Y C S$ transcripts was increased by $55 \%$ in the LP group compared with the CO group. Between the HP and the $\mathrm{CO}$ groups at dpn1 gene expression differed at $P=0.079$ (29\% increase in the HP group compared with the CO group). In contrast, the differences were no longer obvious at weaning age (dpn28) and at dpn185 between offspring of the three maternal diet groups. With regard to the ontogenetic development, the amount of CYCS transcript decreased significantly between the intra-uterine stage at dpn94 and day 1 after birth. Subsequently, CYCS is expressed at a low level at dpn28 and dpn185 (Fig. 4).

\section{Effect of maternal dietary protein level during gestation on methylation of CYCS promoter}

Gene expression data of newborn piglets (dpn1) showed significant differences among the CO, HP and LP groups. Thus, we isolated DNA from newborn piglets (dpn1) for methylation analysis. The level of methylation of individual CpG sites of CYCS at dpn1 is shown in Fig. 5. In total, forty-seven CpG sites from the nt position -417 to -10 were investigated. Significant differences between the CO and LP groups were found for fourteen $\mathrm{CpG}$ sites at positions nt $-253,-224$, $-195,-191,-160,-158,-156,-97,-84,-79,-67$ and $-57(P<0.05)$. Differences between the CO and HP groups were significant for eight $\mathrm{CpG}$ sites at positions $\mathrm{nt}$ $-253,-224,-195,-158,-140,-84,-79$ and -75 $(P<0.05)$. Position nt -140 showed a decrease in methylation level in the LP group in comparison with the CO group at $P=0.086$. The mean of the methylation level over the $\mathrm{CpG}$ sites from nt -417 to -10 is significantly decreased in the LP $(P=0.007)$ and HP ( $P=0.009)$ groups compared with the $\mathrm{CO}$ group. The mean level of methylation of the $\mathrm{CpG}$ sites that differed between the LP and CO groups correlated negatively with transcript abundance $(R-0.419)$ at $P=0.084$. Also, the mean levels of methylation of the CPG sites differing between the HP and CO groups showed a significant negative relationship between methylation and transcript amount $(R-0 \cdot 501, P=0 \cdot 041)$.

Most of the significant differences in methylation status of single CpG sites were found between nt -253 and the transcription start site $(n t+1)$. Interestingly, the sequence spanning $n t-1$ to -250 is highly conserved between mice, humans, dogs, cows and pigs (Fig. 3). Moreover, there was no evidence of any polymorphism among the porcine samples analysed in the present study.

\section{Discussion}

The influence of maternal nutrition on offspring gene expression and $\mathrm{CpG}$ island promoter methylation has been investigated in several genes mainly in rodent models. To the authors' knowledge, the present results show for the first time that hepatic gene expression of CYCS in offspring is affected by specific CPG nt methylation in CYCS promoter depending on dietary maternal protein level during gestation in the pig. CYCS was investigated in the liver tissue because liver is sensitive to maternal nutrient intake during gestation.

CYCS expression of piglets whose mothers were fed a protein-restricted diet (LP) during gestation was significantly increased in comparison with control piglets at dpn1. In the HP group, the expression of CYCS showed a trend towards increased levels. At day 94 of gestation, dpn28 and dpn185, no differences among the three diet groups were obvious, thus implying no long-term effects of maternal protein level on CYCS gene expression. However, in neonatals, differences with the gestation diets might indicate a different responsiveness to changes associated with the transition from intrauterine to extra-uterine life of offspring derived from nonadequately fed gilts compared with offspring of control sows. Gene expression differences in CYCS caused by intrauterine protein undernourishment suggest an interrelation between prenatal nutrition and the outcome of mitochondrial energy metabolism. Furthermore, there is evidence that oxidative stress is associated with intra-uterine growth retardation ${ }^{(30)}$. Additionally, these results are consistent with data from sheep models of maternal nutrient restriction showing increased cytochrome $c$ protein abundance in the liver of 140-d-old fetuses ${ }^{(31)}$ indicating enhanced mitochondrial activity in offspring from nutrient-restricted mothers. 

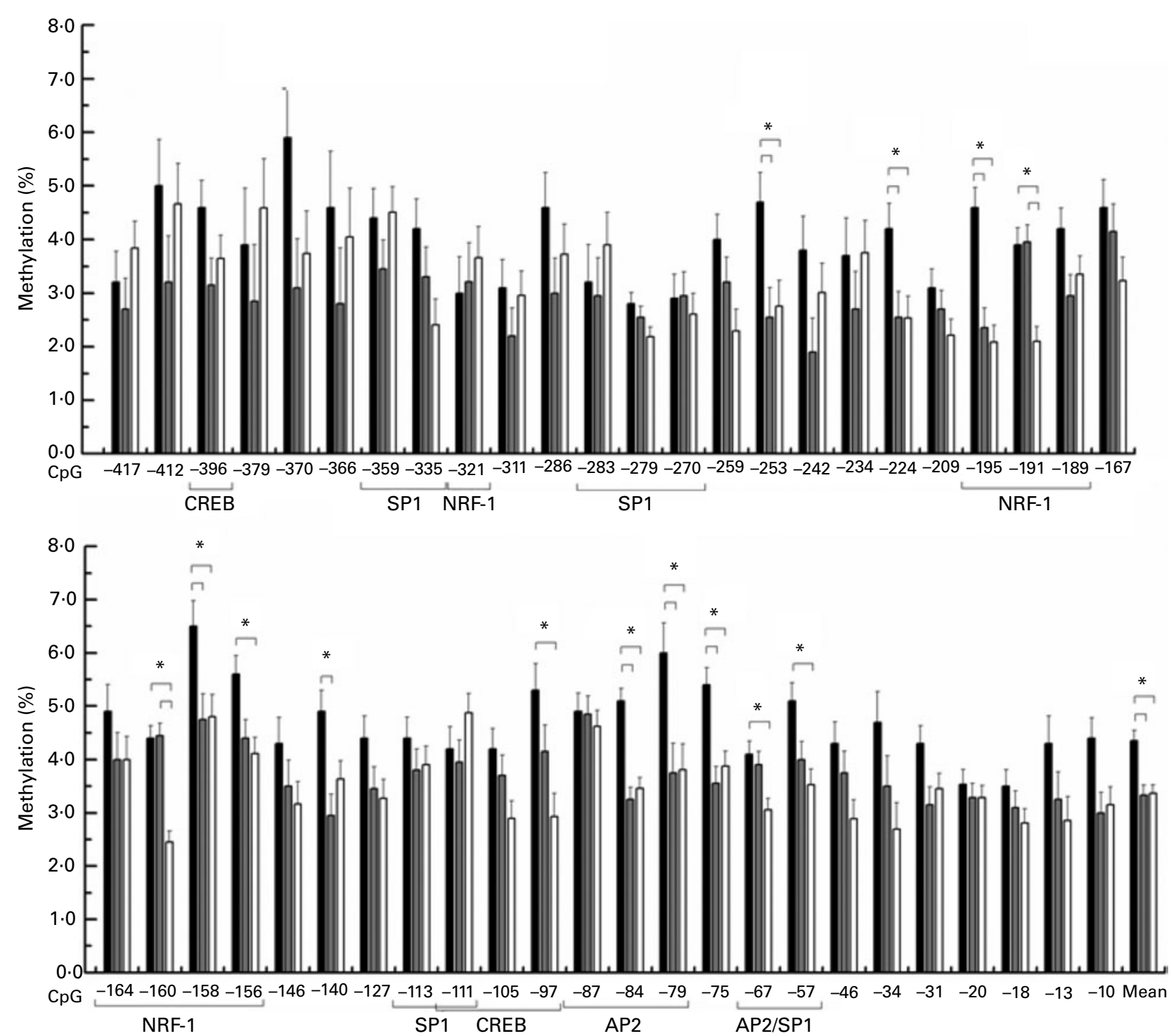

Fig. 5. Methylation of individual $\mathrm{CpG}$ sites in the CYCS promoter region in the liver tissue of pigs from three diet groups at dies post natum (dpn)1. Values are least square means, with their standard errors represented by vertical bars. * Least square means were significantly different $(P<0.05)$. CO ( $\square)$, control; HP ( $\square)$, high protein; LP ( $\square$ ), low protein; NRF-1, nuclear respiratory factor 1; SP1, specificity protein 1; CREB, cAMP response element-binding; AP2, activating protein 2.

Results of gene expression measurement may reflect the different offspring nutritional requirements in the course of pre- and perinatal development. In the last trimester of gestation, fetuses show a tremendous growth and weight gain with increasing protein accretion of up to $4.63 \mathrm{~g} / \mathrm{d}^{(32,33)}$. Thus, during the last period of gestation, fetuses are exceedingly susceptible to maternal protein restriction, developing metabolic adaptations to their nutritional situation. Therefore, we assume that differences in body weight and CYCS expression were not yet obvious at dies post conceptionem 94 since the fetuses were able to cope with maternal protein restriction in the LP group. But at dpn1 differences occur due to high fetal requirements of protein during the last quarter of gestation that could not be supplied in the LP group in contrast to the CO group. Thus, the effects were clearly developed at dpn1. Offspring of the HP group was only slightly affected by the maternal diet during gestation observable in similar birth weight with $\mathrm{CO}^{(24)}$ and showing slight but recognisable differences in CYCS gene expression compared with the CO group.

Simultaneous to the increase in CYCS gene expression in 1-d-old LP piglets, there is a significant increase in methylation of distinct $\mathrm{CpG}$ sites in the promoter region of CYCS in the LP offspring as well as in the HP offspring. The observed methylation level was quite low and varied between 2 and $10 \%$. Nevertheless, there were significant differences in CpG methylation of individual CPG sites of the LP and HP groups in comparison with the CO group. Furthermore, CpG sites showing significantly different methylation levels also exhibited a significant negative correlation with the expression of CYCS, thus suggesting an involvement of DNA methylation of the promoter $\mathrm{CPG}$ island in transcriptional regulation of CYCS.

CpG sites in the $5^{\prime}$-flanking region of porcine CYCS showing that significantly different methylation levels coincide with 
putative TFBS. The location of differentially methylated CpG dinucleotides within putative TFBS indicates a potential regulation of CYCS transcription by DNA methylation. Additionally, most of the significant different methylated CpG sites were found between $n t-253$ and the transcription start site $(n t+1)$ being located in an evolutionary conserved region between mice, humans, dogs, cows and pigs with a conservation identity of $\geq 70 \%$ (Fig. 2). Thus, this region is supposed to be a functional promoter region playing a role in cytochrome $c$ expression control.

In human and rodent cytochrome $c$ promoter recognition sites for nuclear respiratory factor 1 (NRF-1), specific protein 1 and cAMP-response element-binding protein were identified previously ${ }^{(34,35)}$. The discovered NRF-1 site was highly conserved between rats and humans, and is also highly conserved between murine, human, bovine and porcine sequences (Fig. 2). In porcine $5^{\prime}$-flanking region, this conserved NRF-1 site is located at position nt -166 to -153 (Fig. 3) and contains four $\mathrm{CpG}$ sites, of which three show significant differences in methylation level between the CO and LP groups as well as in the HP group (nt $-160,-158,-156$; Fig. 5). These data suggest an influence of DNA methylation at this position on binding of NRF-1. NRF-1 is a transcription factor that is involved in regulation of several genes with mitochondrial respiratory function ${ }^{(36)}$. In silico search for putative NRF-1 binding sites within the porcine 5 -flanking region exhibited two further sites (Fig. 3). Furthermore, two putative cAMP-response element-binding protein sites conserved among pigs, cows and dogs were found, and one of them contained a CpG with significant differences between methylation level of CO and LP (nt - 97; Fig. 5). Additionally, recognition sites of specific protein 1 and activator protein 2 were identified in the porcine sequence. Inhibition of transcription factor binding in specific protein 1 and activator protein 2 binding sites by methylation within and adjacent to the recognition site has been described ${ }^{(37-39)}$

The low variation in the methylation levels $<10 \%$ implicates a sensitive transcriptional control via CpG methylation. These findings correspond with the results reported in the literature $^{(40-42)}$. Thus Lillycrop et al. ${ }^{(40)}$ who found slight changes in PPAR $\alpha$ promoter methylation between 4 and $10 \%$, and thus attached to the indispensability of a precise transcriptional control in phenotype formation during prenatal environment in contrast to gene imprinting with the result of complete gene silencing. Nevertheless, significantly differences between the $\mathrm{CO}$ and LP groups were still observable. Our data suggest that methylation of distinct $\mathrm{CpG}$ sites within the promoter region of CYCS is involved in alteration of its expression caused by prenatal protein restriction.

In summary, we show that gestational protein restriction as well as excess lead to significant differences in DNA methylation level of distinct $\mathrm{CpG}$ sites in $5^{\prime}$-flanking region of CYCS between the offspring of control and the offspring derived from gilts with restricted or excess protein supply during pregnancy. The decrease in methylation level in offspring of LP and HP groups was associated with an increase in CYCS gene expression, implicating an involvement of methylation in the transcriptional control of porcine CYCS in the liver, which seems to allow subtle as well as rapid alterations in gene expression. Interestingly, significant hypomethylation of $\mathrm{CpG}$ sites in the experimental groups was particularly observed at conserved regions that represent putative TFBS. In general, effects of the low-protein diets on expression and DNA methylation of CYCS, a key component of oxidative mitochondrial energy production, as well as fetal growth were more pronounced than the impact of the high-protein diet, indicating that the offspring could better cope with excess rather than restricted protein supply.

\section{Acknowledgements}

S. A. analysed the data and drafted the manuscript; E. M. and M. S. helped in drafting the manuscript; C. C. M. designed the study regarding the nutrition physiological part and collected the material as well as helped in drafting the manuscript; $\mathrm{K}$. W. and S. P. designed the study regarding the molecular genetic part, contributed to data interpretation and helped in drafting the manuscript. The present study was performed within the joint research project FEPROeXPRESS ('Epigenetic mechanisms') within the framework of FUGATOplus and partly supported by grants of the German Federal Ministry of Education and Research (Bundesministerium für Bildung und Forschung, Germany). The authors declare no conflict of interest.

\section{References}

1. Barker DJ, Gluckman PD, Godfrey KM, et al. (1993a) Fetal nutrition and cardiovascular disease in adult life. Lancet 341, 938-941.

2. Barker DJ, Hales CN, Fall CH, et al. (1993b) Type 2 (noninsulin-dependent) diabetes mellitus, hypertension and hyperlipidaemia (syndrome $\mathrm{X}$ ): relation to reduced fetal growth. Diabetologia 36, 62-67.

3. Barker DJ \& Osmond C (1986) Infant mortality, childhood nutrition, and ischaemic heart disease in England and Wales. Lancet i, 1077-1081.

4. Hales CN \& Barker DJ (2001) The thrifty phenotype hypothesis. Br Med Bull 60, 5-20.

5. Wu G, Bazer FW, Wallace JM, et al. (2006) Board-invited review: intrauterine growth retardation: implications for the animal sciences. J Anim Sci 84, 2316-2337.

6. Wu G, Bazer FW, Burghardt RC, et al. (2010) Impacts of amino acid nutrition on pregnancy outcome in pigs: mechanisms and implications for swine production. J Anim Sci 88, E195-E204.

7. Ashworth CJ, Finch AM, Page KR, et al. (2001) Causes and consequences of fetal growth retardation in pigs. Reprod Suppl 58, 233-246.

8. Lucas A (1994) Role of nutritional programming in determining adult morbidity. Arch Dis Child 71, 288-290.

9. Lucas A, Baker BA, Desai M, et al. (1996) Nutrition in pregnant or lactating rats programs lipid metabolism in the offspring. Br J Nutr 76, 605-612.

10. Langley-Evans SC, Bellinger L \& McMullen S (2005) Animal models of programming: early life influences on appetite and feeding behaviour. Matern Child Nutr 1, 142-148.

11. Mortensen OH, Olsen HL, Frandsen L, et al. (2010) Gestational protein restriction in mice has pronounced effects 
on gene expression in newborn offspring's liver and skeletal muscle; protective effect of taurine. Pediatr Res 67, 47-53.

12. Jaenisch R \& Bird A (2003) Epigenetic regulation of gene expression: how the genome integrates intrinsic and environmental signals. Nat Genet 33, Suppl., 245-254.

13. Allis CD, Jenuwein T, Reinberg D, et al. (2007) Epigenetics. New York, NY: Cold Spring Laboratory Press.

14. Santos KF, Mazzola TN \& Carvalho HF (2005) The prima donna of epigenetics: the regulation of gene expression by DNA methylation. Braz J Med Biol Res 38, 1531-1541.

15. Gardiner-Garden $M$ \& Frommer M (1987) CpG islands in vertebrate genomes. J Mol Biol 196, 261-282.

16. McClelland M \& Ivarie R (1982) Asymmetrical distribution of CpG in an 'average' mammalian gene. Nucleic Acids Res 10, $7865-7877$.

17. Cuadrado M, Sacristan M \& Antequera F (2001) Speciesspecific organization of $\mathrm{CpG}$ island promoters at mammalian homologous genes. EMBO Rep 2, 586-592.

18. Mitchell M, Schulz SL, Armstrong DT, et al. (2009) Metabolic and mitochondrial dysfunction in early mouse embryos following maternal dietary protein intervention. Biol Reprod 80, 622-630.

19. Theys N, Bouckenooghe T, Ahn MT, et al. (2009) Maternal low-protein diet alters pancreatic islet mitochondrial function in a sex-specific manner in the adult rat. Am J Physiol Regul Integr Comp Physiol 297, R1516-R1525.

20. Earnshaw WC (1999) Apoptosis. A cellular poison cupboard. Nature 397, 389.

21. Liu X, Kim CN, Yang J, et al. (1996) Induction of apoptotic program in cell-free extracts: requirement for dATP and cytochrome c. Cell 86, 147-157.

22. Kluck RM, Bossy-Wetzel E, Green DR, et al. (1997) The release of cytochrome $c$ from mitochondria: a primary site for Bcl-2 regulation of apoptosis. Science 275, 1132-1136.

23. Zou H, Henzel WJ, Liu X, et al. (1997) Apaf-1, a human protein homologous to $\mathrm{C}$. elegans CED-4, participates in cytochrome $c$-dependent activation of caspase-3. Cell $\mathbf{9 0}$, 405-413.

24. Rehfeldt C, Lang IS, Gors S, et al. (2010) Low and excess dietary protein levels during gestation affect growth and compositional traits in gilts and impair offspring fetal growth. J Anim Sci 89, 329-341.

25. Sarr O, Louveau I, Kalbe C, et al. (2010) Prenatal exposure to maternal low or high protein diets induces modest changes in the adipose tissue proteome of newborn piglets. J Anim Sci 88, 1626-1641.

26. Ovcharenko I, Loots GG, Giardine BM, et al. (2005) Mulan: multiple-sequence local alignment and visualization for studying function and evolution. Genome Res 15, 184-194.

27. Rice P, Longden I \& Bleasby A (2000) EMBOSS: the European Molecular Biology Open Software Suite. Trends Genet 16, 276-277.

28. Vanselow J, Pohland R \& Furbass R (2005) Promoter2-derived Cyp19 expression in bovine granulosa cells coincides with gene-specific DNA hypo-methylation. Mol Cell Endocrinol 15, 57-64.

29. Lewin J, Schmitt AO, Adorjan P, et al. (2004) Quantitative DNA methylation analysis based on four-dye trace data from direct sequencing of PCR amplificates. Bioinformatics 20, 3005-3012.

30. Karowicz-Bilinska A, Suzin J \& Sieroszewski P (2002) Evaluation of oxidative stress indices during treatment in pregnant women with intrauterine growth retardation. Med Sci Monit 8, CR211-CR216.

31. Yakubu DP, Mostyn A, Hyatt MA, et al. (2007) Ontogeny and nutritional programming of mitochondrial proteins in the ovine kidney, liver and lung. Reproduction 134, $823-830$.

32. Kim SW, Hurley WL, Wu G, et al. (2009) Ideal amino acid balance for sows during gestation and lactation. J Anim Sci 87, E123-E132.

33. McPherson RL, Ji F, Wu G, et al. (2004) Growth and compositional changes of fetal tissues in pigs. J Anim Sci 82, 2534-2540.

34. Evans MJ \& Scarpulla RC (1989) Interaction of nuclear factors with multiple sites in the somatic cytochrome $c$ promoter. Characterization of upstream NRF-1, ATF, and intron Sp1 recognition sequences. J Biol Chem 264, $14361-14368$.

35. Vercauteren K, Pasko RA, Gleyzer N, et al. (2006) PGC-1related coactivator: immediate early expression and characterization of a CREB/NRF-1 binding domain associated with cytochrome $c$ promoter occupancy and respiratory growth. Mol Cell Biol 26, 7409-7419.

36. Evans MJ \& Scarpulla RC (1990) NRF-1: a trans-activator of nuclear-encoded respiratory genes in animal cells. Genes Dev 4, 1023-1034.

37. Clark SJ, Harrison J \& Molloy PL (1997) Sp1 binding is inhibited by $(\mathrm{m}) \mathrm{Cp}(\mathrm{m}) \mathrm{CpG}$ methylation. Gene 195, 67-71.

38. Comb M \& Goodman HM (1990) CPG methylation inhibits proenkephalin gene expression and binding of the transcription factor AP-2. Nucleic Acids Res 18, 3975-3982.

39. Zhu WG, Srinivasan K, Dai Z, et al. (2003) Methylation of adjacent $\mathrm{CpG}$ sites affects Sp1/Sp3 binding and activity in the p21(Cip1) promoter. Mol Cell Biol 23, 4056-4065.

40. Lillycrop KA, Phillips ES, Torrens C, et al. (2008) Feeding pregnant rats a protein-restricted diet persistently alters the methylation of specific cytosines in the hepatic PPAR alpha promoter of the offspring. Br J Nutr 100, 278-282.

41. McGowan PO, Sasaki A, D'Alessio AC, et al. (2009) Epigenetic regulation of the glucocorticoid receptor in human brain associates with childhood abuse. Nat Neurosci 12, 342-348.

42. Oberlander TF, Weinberg J, Papsdorf M, et al. (2008) Prenatal exposure to maternal depression, neonatal methylation of human glucocorticoid receptor gene (NR3C1) and infant cortisol stress responses. Epigenetics 3, 97-106. 